\title{
The Four-and-a-Half LIM Domain Protein 2 Supports Influenza A Virus-Induced Lung Inflammation by Restricting the Host Adaptive Immune Response
}

Dörthe Masemann, ${ }^{*}$ Rafael Leite Dantas, ${ }^{*}$ Siarhei Sitnik, ${ }^{*}$ Tanja Schied, ${ }^{*}$ Carolin Nordhoff, ${ }^{*}$ Stephan Ludwig, ${ }^{\dagger \dagger}$ and Viktor Wixler*

From the Institute of Molecular Virology* and the Cluster of Excellence Cells in Motion, ${ }^{\dagger}$ Westfaelische Wilhelms University, Muenster, Germany

Accepted for publication

February 2, 2018.

Address correspondence to Viktor Wixler, Ph.D., Institute of Molecular Virology, Westfaelische Wilhelms University, Von-Esmarch-Str. 56, 48149 Muenster, Germany. E-mail: vwixler@uni-muenster.de.

\begin{abstract}
Four-and-a-half LIM domain protein 2 (FHL2) is a multifunctional adaptor protein with fine-tuning adjustment properties. It acts as a regulator of signaling cascades but also as a cofactor of transcription and controls several anti-inflammatory immune responses. Recently, we described FHL2 as a novel regulator of influenza A virus propagation. We have shown that in vitro FHL2 restricts viral replication by accelerating the interferon regulatory factor 3-dependent transcription of the Ifnb1 gene. In this work, we unraveled an ambiguous role of FHL2 during influenza A virus infection in vivo. Although FHL2 restrained viral replication during the first 24 hours of infection, it significantly delayed viral clearance afterward. Comparison of lung immune status of wild-type and FHL2 knockout mice during influenza virus infection did not acknowledge significant differences in the innate host immune response but revealed an improved migration of dendritic cells from infected lungs into draining lymph nodes as well as increased levels of activated $\mathrm{CD}^{+} \mathrm{T}$ lymphocytes accumulated in the lungs of FHL2 knockout mice. (Am J Pathol 2018, 188: 1236-1245; https://doi.org/10.1016/j.ajpath.2018.02.004)
\end{abstract}

The main task of influenza A viruses (IAVs), like any viral pathogen, is to produce as much progeny as possible. To propagate successfully, IAVs have to enter into a symbiosis with cellular and host intrinsic pathways and activities. By that influenza viruses do not only modulate signaling cascades and transcription and translation in infected cells to benefit immediately after infection, but they also have to circumvent cellular innate and host immune defense barriers. $^{1-3}$ The genome of IAVs codes for at least two nonstructural proteins, nonstructural protein 1 (NS1) and PB1-F2, that suppress cellular and host immunity by interfering with multiple antiviral host signaling cascades. ${ }^{4,5}$ On the other side, influenza virus permissive cells and host organisms developed in the course of evolution are effective antiviral defense mechanisms. Affected cells normally respond to infection by induction of an antiviral state. It is initiated by sensing viral 5'-triphosphate RNA by the intracellular retinoic acid-inducible gene I receptor and subsequent induction of the type I interferon (IFN) response, which is a powerful restriction system for transcription and translation of viral RNA and proteins. ${ }^{6,7}$ To the cellular intrinsic response the host innate and adaptive immune responses follow that complete the IAV infection clearance and support the wound healing, and all together are part of the defense program of the infected organism to protect itself from viral spread and tissue destruction. ${ }^{8,9}$ This highly regulated process of pathogen-host interactions implies involvement of numerous cellular proteins that may either support or suppress viral replication.

\footnotetext{
Supported by Coordination for the Improvement of Higher Education Personnel (CAPES) Brazil grant 99999.013234/2013-07 (R.L.D.), IZKF Muenster grants Wix2/005/10 (V.W.) and Lud2/008/17 (S.L.), and Deutsche Forschungsgemeinschaft grants SFB 492 A17 (V.W.) and SFB 1009 B02 (S.L.).
}

Disclosures: None declared. 
The four-and-a-half LIM domain protein 2 (FHL2) is a multifunctional intracellular adaptor protein that is involved in modulation of numerous cellular events, including proinflammatory and anti-inflammatory activities. ${ }^{10,11}$ By binding to transmembrane receptors and structural proteins, kinases, or transcription factors, FHL2 executes fine-tuning adjustments of signaling cascades and gene transcription activities. It adjusts expression of extracellular matrix proteins and matrix metalloproteinases, cytoskeletal proteins, and cytokines, but also migration of fibroblasts and dendritic cells (DCs), as well as functional activities of myofibroblasts, natural killer (NK) cells, osteoclasts, and macrophages. ${ }^{12-17}$ Consequently, deficiency of FHL2 affects wound healing and several chronic immune diseases. ${ }^{17-19}$

Previously, we showed that FHL2 acts as a cell intrinsic negative regulator of IAV replication by positively regulating IFN- $\beta$ expression by IFN regulatory factor 3 (IRF-3)-dependent mechanisms. ${ }^{20}$ Consequently, IAVs replicated more efficiently in FHL2-deficient cells than in cells expressing the protein. Considering that FHL2 also effects certain immune cell populations, we investigated the influence of FHL2 in vivo on the lung immune response against IAV infection. Here, we demonstrate that contrary to our expectation, deficiency in FHL2 leads to faster overall clearance of IAV infection in vivo. FHL2 knockout $\left(\mathrm{FHL} 2^{\mathrm{KO}}\right.$ ) mice showed increased lung expression of the chemokine (C-C motif) ligand 19 (CCL19) and the sphingosine 1-phosphate receptor 1, known to highly affect DC migration. ${ }^{15}$ Accordingly, mice absent of FHL2 exhibited accelerated migration of DCs into lung regional lymph nodes (LNs) on IAV infection and enhanced CD8 ${ }^{+}$ T-lymphocyte recruitment to the site of infection. Thus, our data emphasize the ambivalent role of FHL2 in the antiviral immune response in vivo. Although it induced an intracellular antiviral state by supporting the IFN type I immune response at early time points, it restricted migration of DCs from the site of infection into regional LNs, thus limiting activation and recruitment of $\mathrm{T}$ lymphocytes of the adaptive immune response and decreasing viral clearance in vivo.

\section{Materials and Methods}

\section{Animal Studies}

All animal studies were performed in compliance with the German regulations of the Society for Laboratory Animal Science and the European Health Law of the Federation of Laboratory Animal Science Associations and were approved by the Landesamt für Natur, Umwelt und Verbraucherschutz Nordrhein-Westfalen, Germany. All experiments were performed with 2- to 6-month-old $F H L 2^{+/+}$or $F H L 2^{-/-}$mice ${ }^{21}$ of a $\mathrm{C} 57 \mathrm{Bl} / 6$ genetic background housed under pathogen-free conditions.

Before infection, mice were anesthetized by inhalation of $2.5 \%$ isoflurane (Baxter, Deerfield, IL), weighed, and infected by intranasal administration of $50 \mu \mathrm{L}$ of viral stock solution of influenza virus PR8 [A/Puerto Rico/8/34
(H1N1)] in phosphate-buffered saline. A total amount of 300 plaque-forming units per animal was instilled, and the health status of infected animals and body weight loss were monitored daily. The number of infectious virus particles in the bronchoalveolar lavage (BAL) fluid (BALF) was determined by standard plaque assay as previously described, ${ }^{22}$ and viral titers were expressed as plaqueforming units per milliliter.

\section{RNA Isolation, Reverse Transcription, and RT-qPCR}

Mouse lungs were collected at indicated time points, and total lung RNA was isolated with TRIzol Reagent (Thermo Fisher Scientific, Darmstadt, Germany). Half of each lung was homogenized (FastPrep-24 homogenizator; MP Biomedicals, Eschwege, Germany), and the RNA was precipitated with isopropanol. The precipitated RNA was further purified in a secondary phase separation step as described previously. ${ }^{23}$ Purified RNA was transcribed into cDNA with the use of the high-capacity cDNA reverse transcription kit from Applied Biosystems (Darmstadt, Germany). mRNA expression levels were determined by TaqMan quantitative real-time RT-PCR (RT-qPCR) with the use of the LightCycler 480 II (Roche Diagnostics, Mannheim, Germany). Each cDNA sample was analyzed in triplicate, and specific signals were scored in relation to the signal of the housekeeping gene transcript cytochrome $c(C y c s)$. For analysis of murine mRNA expression levels the following primer pairs were used: 5'-ATTTCTACAGCCCCCAGAGC-3' (forward) and $5^{\prime}$-TGGTATTCTCGCCGATGTAGT-3' (reverse) for CC chemokine receptor 7 (CCR7; Ccr7); 5'-TCCAAGGGCTGCAAGAGA-3' (forward) and 5'-TGAAGTTCGTGGGGGATCT-3' (reverse) for CCL21 (Ccl21c); 5'-TGTGGCCTGCCTCAGATTAT-3' (forward) and 5'-AGTCTTCCGCATCATTAGCAC-3' (reverse) for CCL19 (Ccl19); 5'CGGTGTAGACCCAGAGTCCT-3' (forward) and 5'-AGCTTTTCCTTGGCTGGAG-3' (reverse) for sphingosine 1phosphate receptor 1 (S1PR1; S1prl); 5'-GCTACCCATGGTCTCATCGT- $3^{\prime}$ (forward) and $5^{\prime}$-GAAACCCCTCCGAATGCT-3' (reverse) for Cycs, and for analysis of viral mRNA: 5' - GAGCATCCGTCGGAAAAAT-3' (forward) and 5'-CGGTGCACATTTGGATGTAG-3' (reverse) for nucleoprotein (NP; NP); 5'-GGCAGTACTCTCGGTCTGGA-3' (forward) and 5'-CCGCTCCACTATCTGCTTTC-3' (reverse) for NS1 (NS1).

\section{Immunohistochemistry}

Half of the lung of each mouse was isolated, degassed, and fixed in $4 \%$ paraformaldehyde for 5 to 7 hours at room temperature, dehydrated in ascending isopropanol dilutions, and embedded in paraffin. Paraffin sections of $4 \mu \mathrm{m}$ were analyzed. Lung sections were dewaxed and rehydrated, and antigens were demasked by heating the samples in $10 \mathrm{mmol} /$ $\mathrm{L}$ citric acid buffer, $\mathrm{pH} 6.0$, at $100^{\circ} \mathrm{C}$ for 20 minutes. Afterward paraffin sections were blocked with $10 \%$ fetal 
bovine serum, containing $0.1 \%$ Triton $\mathrm{X}-100$ for 30 minutes. Specific staining of influenza virus NP was performed by incubation with goat anti-influenza NP antibody (clone G150) [dilution 1:2500; kind gift of Dr. Robert Webster (St. Jude Children's Research Hospital, Memphis, $\mathrm{TN}$ )] for 1 hour at room temperature, followed by speciesspecific secondary antibody incubation for 30 minutes. The Vectastain ABC-AP Kit (Vector Laboratories, Dossenheim, Germany) was used for visualization according to the manufacturer's protocol.

\section{Analysis of Lung Immune Status}

BAL was performed as previously described. ${ }^{24}$ Briefly, mice were euthanized, the trachea was exposed, and the lungs were washed three times with $1 \mathrm{~mL}$ of phosphatebuffered saline that contained $2 \mathrm{mmol} / \mathrm{L}$ EDTA for each wash. Supernatants of the first lavage were kept separately for viral plaque titration, lactate dehydrogenase assay, and analysis of cytokine quantities in BALF. Cell pellets of the first lavage were combined with cells of the following lavages and centrifuged at $400 \times g$ for 10 minutes at $4^{\circ} \mathrm{C}$. Erythrocytes were lyzed, cell numbers were determined, and equal amounts of cells were subsequently stained for flow cytometric analysis (Beckman Coulter, Gallios; BD, FACSCalibur, Heidelberg, Germany). For analysis of DCs migrated from lung tissue into regional LNs, mediastinal LNs were isolated at indicated time points and minced through a $45-\mu \mathrm{m}$ cell strainer to generate single-cell suspensions. Afterward, erythrocytes were lyzed, cell numbers were determined, and equal amounts of cells were subsequently stained for flow cytometric analysis (Beckman Coulter, Gallios, Krefeld, Germany).

Before specific staining all samples were incubated with anti-Fc $\gamma$ RII/III (BD Pharmingen, San Diego, CA) to block nonspecific binding of antibodies. Analysis of distinct immune cell populations was performed with flow cytometric antibodies from eBioscience (Frankfurt, Germany) and BioLegend (Fell, Germany) routinely used at concentrations of $1 \mu \mathrm{g} / \mathrm{mL}$ each. Before intracellular staining, $2 \times 10^{5}$ cells/ $500 \mu \mathrm{L}$ were stimulated with $1 \mu \mathrm{L}$ of cell stimulation cocktail with inhibitors (contains ionomycin and transport inhibitors) from eBioscience (reference number 00-4975-93) and incubated for 4 hours at $37^{\circ} \mathrm{C}$. Finally, the cell surface receptors were stained, and the cells were fixed and permeabilized according to the instruction of the Foxp3/Transcription Factor Staining Kit from eBioscience. A detailed list of antibodies used is presented in Table 1.

\section{Determination of Cytokine Levels in BALF}

The quantity of cytokines present in the BALF was analyzed in the first wash of lavage by the LEGENDplex flow-based 13-plex mouse inflammation panel kit (catalog number 740150; BioLegend) according to the manufacturer's protocol.
Table 1 List of Antibodies Used for Flow Cytometric Studies

\begin{tabular}{lllll}
\hline Antigen & Dye & Clone & $\begin{array}{l}\text { Catalog } \\
\text { No. }\end{array}$ & Company \\
\hline CD103 & APC & 2 E7 & 121414 & BioLegend \\
CD11b & BV510 & M1/70 & 101245 & BioLegend \\
CD11b & FITC & M1/70 & 101206 & BioLegend \\
CD11c & Pacific Blue & N418 & 117321 & BioLegend \\
CD11c & PE/Cy7 & N418 & 117318 & BioLegend \\
CD170 & PE & 1RNM44N & $12-1702$ & eBioscience \\
$\quad$ Siglec-F) & & & & \\
CD3e & PE & $145-2 C 11$ & 100308 & BioLegend \\
CD4 & APC & GK1.5 & 100412 & BioLegend \\
CD45.2 & FITC & 104 & 109806 & BioLegend \\
CD49b & FITC & DX5 & 108905 & BioLegend \\
CD8 & FITC & $53-6.7$ & 100706 & BioLegend \\
F4/80 & PerCP/Cy5.5 & BM8 & 123128 & BioLegend \\
IFN- $\gamma$ & APC & XMG1.2 & 554413 & BD Biosciences \\
Ly6G & APC/Cy7 & 1 A8 & 127623 & BioLegend \\
MHCII & PE/Cy7 & M5/114.15.2 & $25-5321$ & eBioscience \\
\hline
\end{tabular}

APC, allophycocyanin; Cy, cyanine; FITC, fluorescein isothiocyanate; IFN, interferon; MHCII, major histocompatibility complex II; PE, phycoerythrin; PerCP, peridinin chlorophyll protein.

\section{Statistical Analysis}

Data are expressed as the means \pm SEM. Statistical analysis was performed with the $U$-test and GraphPad Prism software version 7.0 (GraphPad, San Diego, CA). Results were considered statistically significant at $P<0.05$.

\section{Results}

Recently, it was shown that FHL2 improved the cellular innate immune response to IAV infection by enhancing the IRF-3-dependent transcription of the IFN- $\beta$ (Ifnbl) gene. ${ }^{20}$ Consequently, in vitro, IAVs replicated significantly more efficiently in FHL2-deficient than in wild-type (WT) cells. To assess whether the IFN-supporting property of FHL2 was also an advantage in restraining influenza virus replication in vivo, $F H L 2^{W T}$ and knockout $\left(F H L 2^{K O}\right)$ mice were infected with IAV PR8, and progeny virus titers in their lungs were compared. After intranasal inoculation of 300 plaque-forming units, representing approximately $1 \times \mathrm{LD}_{50}$ for $\mathrm{C} 57 \mathrm{Bl} / 6 \mathrm{WT}$ mice, BALFs of mice were obtained 1,3 , or 7 days after infection, and virus titers were determined. One day after infection, lungs of $F H L 2^{K O}$ mice exhibited significantly increased numbers of viral particles compared with WT mice (Figure 1A and Supplemental Figure S1), which was fully consistent with our previous in vitro data. ${ }^{20}$ However, virus replication was surprisingly impaired at later stages of infection (Figure 1A and Supplemental Figure S1). Already on day 3 after infection, viral replication was increased in the lungs of $F H L 2^{K O}$ mice compared with WT mice. The trend toward beneficial replication of viruses in WT mice increased over time, showing a fivefold excess over $F H L 2^{K O}$ mice on day 7 after 
A

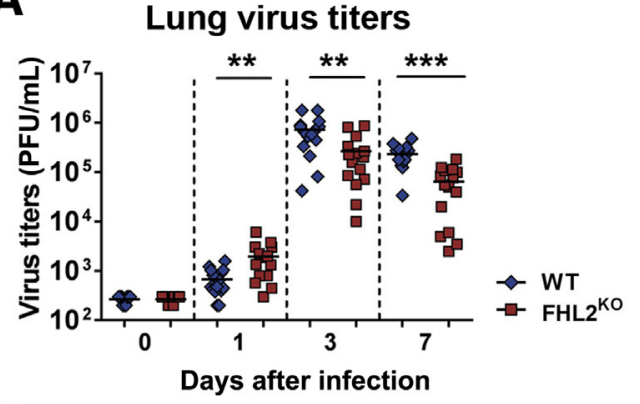

B

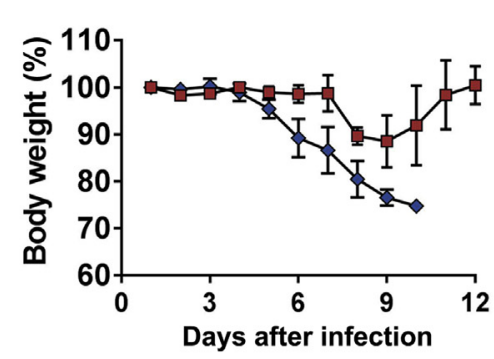

Survival

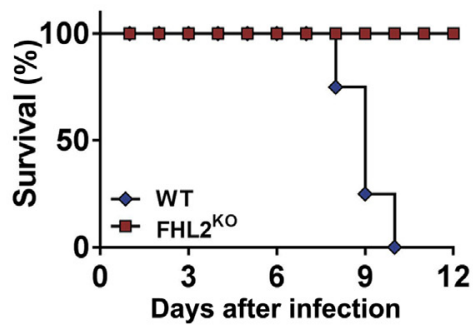

C

Viral NP mRNA

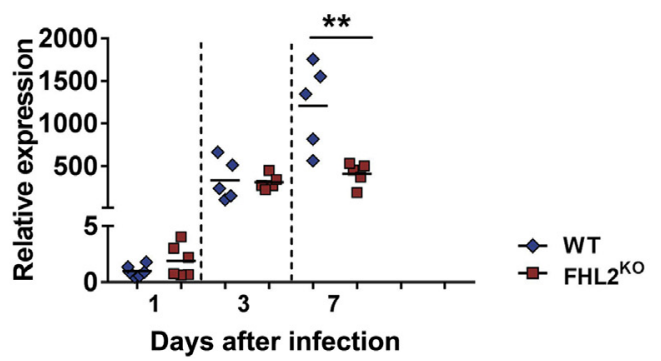

D viral NP protein
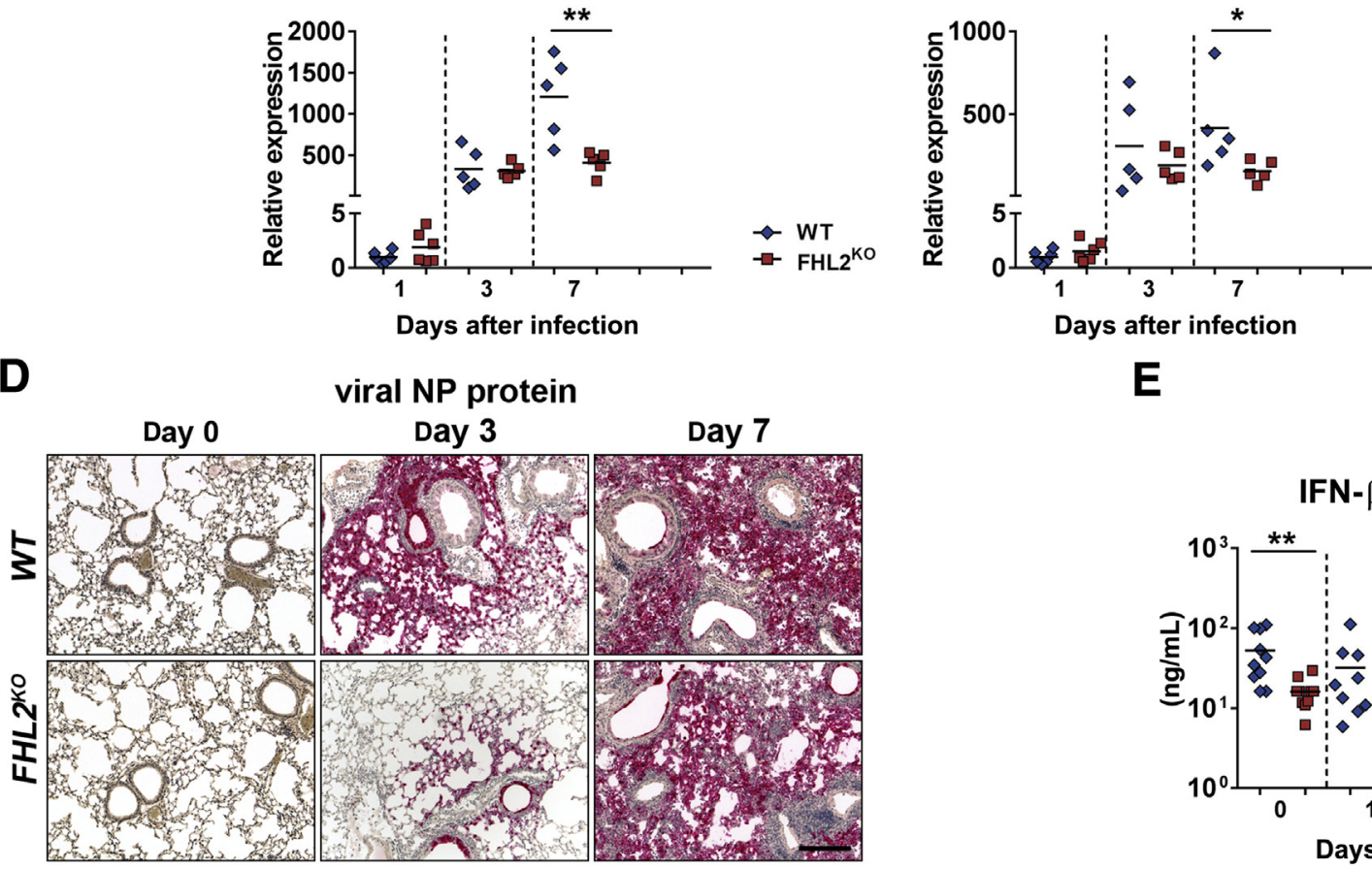

E

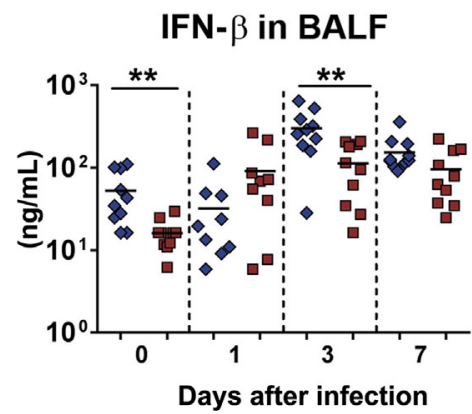

Figure 1 Four-and-a-half LIM domain protein 2 (FHL2) deficiency is beneficial for influenza A virus lung replication in the short run but disadvantageous over the long term. A: Wild-type (WT) and FHL2 knockout $\left(F H L 2^{K O}\right)$ mice were infected with 300 plaque-forming units (PFU; $\left.1 \times L_{50}\right)$ of influenza A/PR/8/34 rec $(P R 8)$ H1N1 strain and at indicated times, virus titers in bronchoalveolar lavage fluid (BALF) were determined by standard plaque assay. Results of separate experiments are shown in Supplemental Figure S1. B: Body weight and survival of PR8-infected WT and $F H L 2^{K O}$ mice were determined daily. Although all FHL $2^{\mathrm{KO}}$ mice recovered, WT mice were sacrificed at day 8 to 10 after infection because of ethical criteria. C: Alterations in viral mRNA expression in lungs during infection. Total lung RNA of noninfected and PR8-infected WT and $F H L 2^{K O}$ mice was isolated. To ensure that only newly synthesized viral mRNA but not viral vRNA or viral cRNA will be recorded, the total lung RNA was transcribed into CDNA using oligo(dT) primers. The expression of viral nucleoprotein (NP) and nonstructural protein 1 (NS1) mRNA was then analyzed by TaqMan quantitative PCR with target-specific primers. D: Paraffin lung sections of WT and $F H L 2^{K O}$ mice infected 0,3 , and 7 days with PR8 virus were stained for expression of viral NP protein (red) and counterstained with hematoxylin (blue). E: The quantity of interferon (IFN)- $\beta$ cytokine in BALF of noninfected and influenza A virus-infected mice was detected by LEGENDplex flow-based 13-plex mouse inflammation panel kit (BioLegend). Values of individual animals and mean values (black lines) per group are shown (A, C, and $\mathbf{E}) . n=3$ independent experiments $(\mathbf{A}) ; n=2$ independent experiments (E). Statistical analysis was performed with the $U$-test. ${ }^{*} P<0.05,{ }^{*} P<0.01$, and ${ }^{* * *} P<0.001$. Scale bar $=200 \mu \mathrm{m}$ (D).

infection. The general kinetics of IAV replication was similar in both mouse strains and did not differ from the classic IAV progression, ${ }^{25,26}$ increasing until day 3 after infection and declining afterward. The improved clearance of influenza viral infection in FHL2-deficient mice was reflected by reduced body weight loss and increased survival. Although all infected $F H L 2^{K O}$ mice recovered until day 12 after infection, all WT mice continuously lost body weight and had to be sacrificed for ethical reasons on day 8 to 10 after infection (Figure 1B).
Examination of mouse lungs for expression of newly synthesized influenza virus mRNA or protein by RT-qPCR or immunohistochemistry confirmed the advanced viral clearance by $F H L 2^{K O}$ mice (Figure 1, C and D). To ensure that only newly synthesized viral mRNA but not viral RNA or viral cRNA were recorded, the total lung RNA was transcribed into cDNA with the use of oligo(dT) primers. On day 1 after infection, no viral proteins could be detected by immunohistochemistry, and only small quantities of viral $N P$ and NS1 mRNA were measurable. Although 
nonsignificant, slightly increased levels of viral mRNA were seen in $F H L 2^{K O}$ mice on day after infection compared with WT mice. With increasing time of infection, reduced viral mRNA expression (Figure 1C) and viral protein load (Figure 1D) were detected in the lungs of FHL2-deficient mice.

Of interest, the quantity of lung IFN- $\beta$ protein in BALF of noninfected WT and $F H L 2^{K O}$ animals reflected the situation observed in vitro. Significantly higher levels were detected in the BALF of WT mice compared with $F H L 2^{K O}$ mice. After infection, the IFN- $\beta$ protein amount gradually increased, reflecting the changes in viral load of lungs (Figure 1E). Measurement of major proinflammatory cytokines in BALF such as IFN- $\gamma$, IL-6, or tumor necrosis factor $\alpha$ that are usually up-regulated during influenza infection, although reflecting the severity of ongoing inflammation, also showed gradually increasing levels after infection, but no remarkable differences between WT and $F H L 2^{K O}$ mice were registered (Supplemental Figure S2).

Thus, the cell intrinsic antiviral function of FHL2 unraveled in the in vitro infection experiments ${ }^{20}$ was detectable in vivo only early after infection and turned into a predominantly virus-supportive action at later times, resulting in decreased clearance of viral infection.

The host innate and adaptive immune responses are important factors that restrict viral replication and clearance. To study whether the switch from a virus-supportive to an antiviral response in $F H L 2^{K O}$ mice compared with WT
A

Total cell number
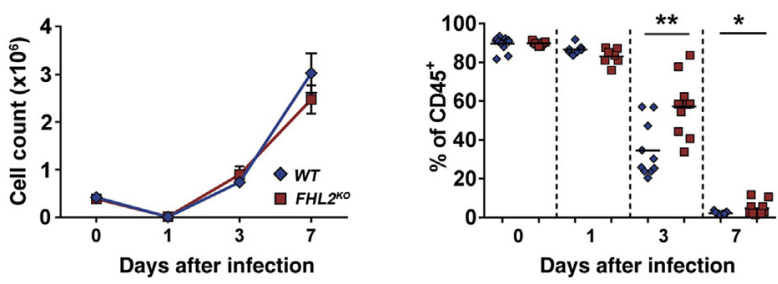

E

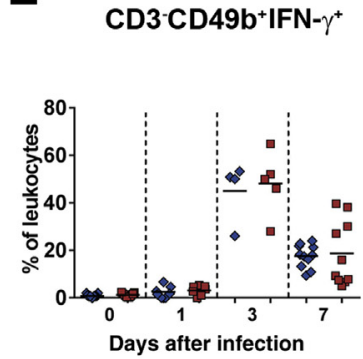

G
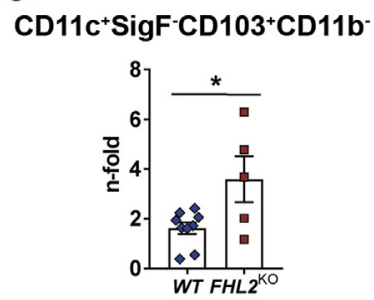

B

$\mathrm{CD}_{11 \mathrm{c}^{+}} \operatorname{SigF}^{+}(\mathrm{aM} \Phi)$

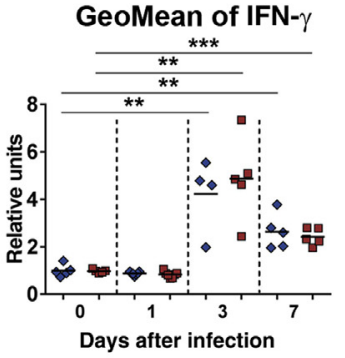

C

$\mathrm{CD}^{11 \mathrm{~b}}{ }^{+} \mathrm{F} 4 / 80^{+}(\mathrm{pM} \Phi)$

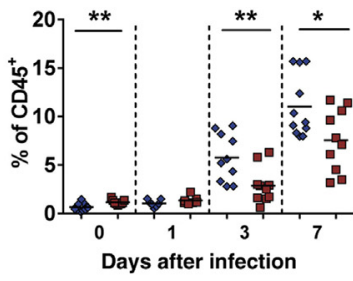

F
CD103+CD11b

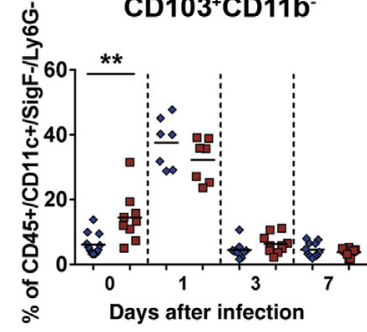

H

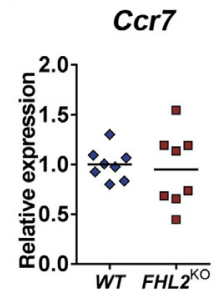

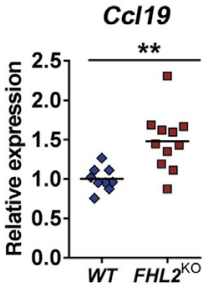

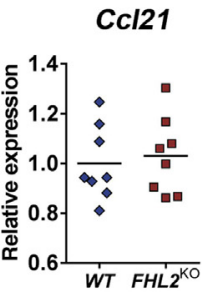

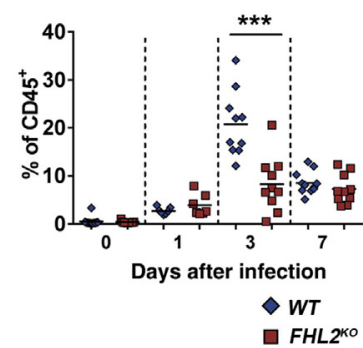

D

\section{CD11b+Ly6G+(neutro)}

$\square$ FHL2 ${ }^{\text {KO }}$

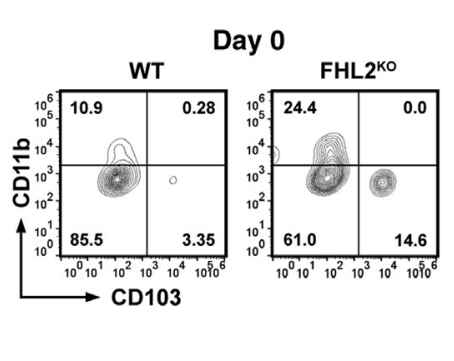

I

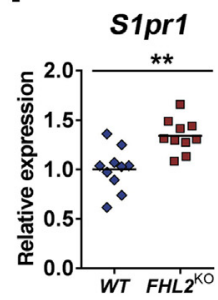

Figure 2 Innate immune status of the bronchoalveolar space of wild-type (WT) and four-and-a-half LIM domain protein 2 knockout (FHL2 ${ }^{K O}$ ) mice after infection with PR8 influenza A virus. A-F: WT and $F H L 2^{K O}$ mice were infected with 300 plaque-forming units of influenza A PR8 virus strain, and bronchoalveolar lavage fluid (BALF) was analyzed at indicated times for the amount of residing cells (A) and for the presence of alveolar macrophages (aM $\Phi$; $\mathbf{B}$ ), peripheral macrophages $(\mathrm{pM} \Phi ; \mathbf{C})$, neutrophils $(\mathbf{D})$, natural killer $(\mathrm{NK})$ cells $(\mathbf{E})$, and dendritic cells $(\mathrm{DCs} ; \mathbf{F})$ by flow cytometry. The right image in $\mathbf{E}$ shows the relative geometric mean fluorescence intensity of intracellularly expressed interferon (IFN) $\gamma$. Right images in $\mathbf{F}$ show representative dot plots that emphasize the gating strategy for quantification of $\mathrm{CD} 103^{+} \mathrm{CD} 11 \mathrm{~b}^{-} \mathrm{DCs}$. G: Migration of $\mathrm{DCs}$ into lung-draining lymph nodes. Mediastinal lymph nodes of noninfected or

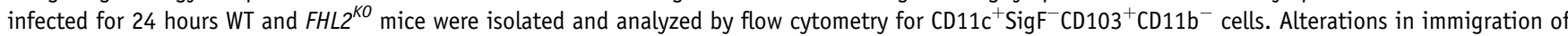
DCs in relation to uninfected mice are shown. $\mathbf{H}$ and I: The expression of Ccr7, Ccl19, Ccl21, and S1pr1 genes in noninfected lungs of WT and FHL2 ${ }^{K O}$ mice was analyzed by TaqMan quantitative real-time RT-PCR. Values of individual animals and mean values (black lines) per group are shown. Statistical analysis was performed with the $U$-test. ${ }^{*} P<0.05,{ }^{* *} P<0.01$, and ${ }^{* * *} P<0.001$. 
animals might be initiated by differentially regulated immune responses, BALF of both mouse strains were analyzed for alterations in the cell immune status during IAV infection. First, changes in the total number of cells presented in BALF were analyzed. Their numbers, the majority of which $(>95 \%)$ were $\mathrm{CD} 45^{+}$, increased with infection but did not significantly differ between the two mouse strains (Figure 2A). Therefore, percentages of distinct immune cell subpopulations were used for further analyses. The main set of lung-resident immune cells, usually presented in lungs of mice and responsible for maintaining the lung tissue homeostasis, is represented by alveolar macrophages. ${ }^{9,27}$ The relative amount of these $\mathrm{SigG}^{+}$and $\mathrm{CD}_{11 \mathrm{~b}^{-}}$but $\mathrm{CD}_{11 \mathrm{c}^{+}}$lung-resident macrophages did not differ between uninfected WT and $F H L 2^{K O}$ mice but, as expected, declined after influenza virus infection because of IAV-induced apoptosis. ${ }^{28}$ In accordance with higher lung viral titers at days 3 and 7 in WT mice, they showed a higher loss of alveolar macrophages than FHL2 ${ }^{K O}$ mice (Figure 2B). The number of $\mathrm{CD} 11 \mathrm{~b}^{+} \mathrm{F} 4 / 80^{+}$ peripheral macrophages was slightly elevated in uninfected $F H L 2^{K O}$ lungs compared with WT lungs. Their amount increased after infection in both mouse strains, but slower in $F H L 2^{K O}$ lungs than in their WT counterparts (Figure 2C). BALFs of WT mice contained on day 3 after infection 8.6 times more peripheral macrophages and on day 7 after infection 11.0 times more peripheral macrophages than uninfected mice. In $F H L 2^{K O}$ mice this increase was only 2.5- and 6.6-fold, respectively. In addition, neutrophils (represented by $\mathrm{CD} 11 \mathrm{~b}^{+} \mathrm{Ly}_{6 \mathrm{G}}{ }^{+}$cells), being only marginally present in the BALF of uninfected mice, showed increased migration into lungs on infection (Figure 2D). On day 3 of infection, BALF of WT mice contained a higher percentage of these cells than $F H L 2^{K O}$ mice, but on day 7 after infection their numbers were equalized. The number of NK cells (represented by $\mathrm{CD} 103^{-} \mathrm{CD} 49 \mathrm{~b}^{+} \mathrm{IFN}-\gamma^{+}$cells) as well as the mean amount of IFN- $\gamma$ per cell (representing the activation of these cells) were also increased in lungs of both mouse strains after infection, particularly on day 3, but to similar extents (Figure 2E). Thus, the innate host immune response represented by macrophages, neutrophils, and NK cells seems not to be responsible for the relative attenuation of viral replication seen on day 3 and 7 after infection in $F H L 2^{K O}$ in comparison with WT lungs (Figure 1A). The induction of innate immune response subpopulations tended to be even weaker in $F H L 2^{K O}$ mice.

For efficient replication, influenza viruses must overcome not only the rather unspecific cellular intrinsic and host innate immune defenses but also the highly specific host adaptive immune response. This response develops only after infection and raises gradually with time, unfolding its maximal strength when innate immune responses are already declining. ${ }^{25,26}$ Nonetheless, it is absolutely crucial for the final IAV clearance and is initiated by antigenpresenting DCs. The main class of lung DCs that are involved in early viral antigen-presentation are

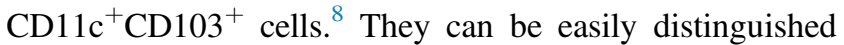
from other innate immune cell populations, such as alveolar macrophages or peripheral macrophages, by the absence of both SigF and CD11b markers on their surface. ${ }^{8,29}$ Analysis of CD11 $\mathrm{c}^{+} \mathrm{CD}_{103}{ }^{+} \mathrm{SigF}^{-} \mathrm{CD} 11 \mathrm{~b}^{-}$DCs in infected WT and $F H L 2^{K O}$ mice showed that their levels raised already on day 1 of infection in BALF of both mouse strains and dropped down before day 3 after infection, indicating their efficient emigration from the lungs (Figure 2F). Of interest, compared with other innate immune cell populations, the levels of $\mathrm{CD} 11 \mathrm{c}^{+} \mathrm{CD} 103^{+} \mathrm{CD} 11 \mathrm{~b}^{-}$DCs was already strikingly increased in steady-state lungs of $\mathrm{FHL}_{2}{ }^{\mathrm{KO}}$ mice compared with WT mice (Figure 2F). Because the $\mathrm{CD} 11 \mathrm{c}^{+} \mathrm{CD} 103^{+} \mathrm{CD} 11 \mathrm{~b}^{-}$DCs are the main subset of lung $\mathrm{DCs}$ that present viral antigens to $\mathrm{T}$ lymphocytes in the draining LNs in the early phase of infection, it was tested whether their migration to the LNs was more efficient in $F H L 2^{K O}$ mice than in WT mice. FHL2 ${ }^{K O}$ and WT mice were infected with 300 plaque-forming units of PR8 virus, and the increase of $\mathrm{CD} 11 \mathrm{c}^{+} \mathrm{CD} 103^{+} \mathrm{CD} 11 \mathrm{~b}^{-}$cells in the mediastinal LNs was analyzed by flow cytometry 24 hours later in relation to noninfected mice. This time point was chosen according to previous publications on DC migration from the lung into regional LNs after influenza virus infection. ${ }^{30}$ Twenty-four hours after PR8 infection there was a twofold higher percentage of antigen-presenting DCs in the mediastinal LNs of $F H L 2^{K O}$ mice than of WT mice (Figure 2G).

Migration of DCs from infected tissues toward regional LNs critically depended on CCR7 and its ligands, CCL19 and CCL21. ${ }^{31}$ To explore whether their expression was changed in FHL2-deficient mice, lung lysates of noninfected mice were analyzed by RT-qPCR. The amount of $\mathrm{Ccr} 7$ and Ccl21 transcripts were not changed in $F H L 2^{K O}$ compared with WT mice, but the amount of Ccll9 mRNA was significantly up-regulated in $F H L 2^{K O}$ lungs (Figure $2 \mathrm{H}$ ). Recently, in in vitro experiments König et $\mathrm{al}^{15}$ showed that FHL2 regulates the expression of the S1PR1 receptor. A coordinated function of S1PR1 with CCR7 was important for activation of Rho GTPases, which in turn play a critical role in migration of DCs. Hence, S1PR1 expression was examined in $F H L 2^{K O}$ mice and compared with WT mice. The experiment confirmed the increased expression of S1PR1 in FHL2-deficient lungs (Figure 2I).

It is well known that influenza viruses generally induce a strong adaptive immune response because of their high antigenicity. ${ }^{32}$ Because increased levels of $\mathrm{CD} 11 \mathrm{c}^{+} \mathrm{CD} 103^{+} \mathrm{CD} 11 \mathrm{~b}^{-}$ DCs were found in lungs of $F H L 2^{K O}$ mice and a more rapid migration of these cells on early time points of IAV infection was observed, it was tested whether WT and $F H L 2^{K O}$ mice differ in their T-lymphocyte response to influenza virus infection. Analysis of $\mathrm{CD}^{+}$and $\mathrm{CD} 8^{+}$cells showed that the percentage of $\mathrm{CD}^{+}{ }^{+} \mathrm{T}$ helper cells in BALF decreased with infection and the level of cytotoxic T cells increased (Figure 3, A and B and Supplemental Figure S3). Of note, the percentage of $\mathrm{CD}^{+}$and the percentage of activated $\mathrm{CD} 8^{+} \mathrm{IFN}-\gamma^{+} \mathrm{T}$ 


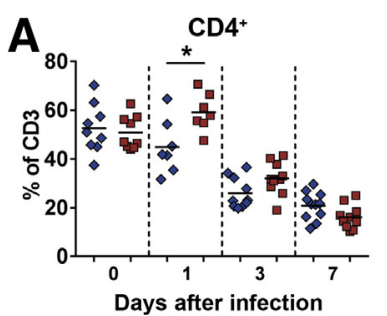

B

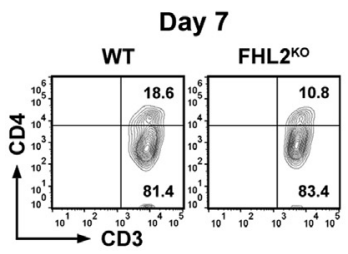

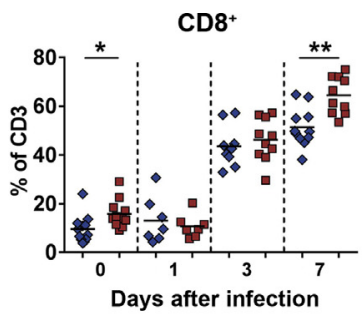

Day 7

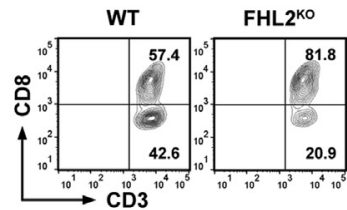

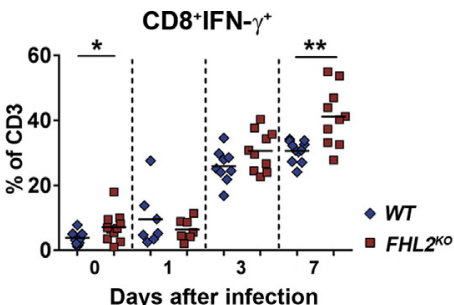

Days after infection

C

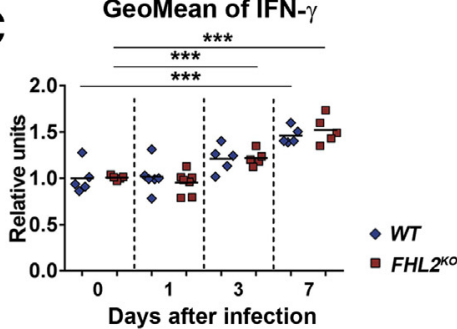

Figure 3 Adaptive immune status of the bronchoalveolar lavage fluid (BALF) of wild-type (WT) and four-and-a-half LIM domain protein 2 knockout $\left(F H L 2^{K O}\right)$ mice after infection with PR8 influenza A virus (IAV). A: WT and $F H L 2^{K O}$ mice were infected with 300 plaque-forming units of influenza A PR8 virus strain, and BALF was analyzed at indicated times for the presence of $\mathrm{CD}_{3}^{+} \mathrm{CD}^{+}, \mathrm{CD}^{+} \mathrm{CD} 8^{+}$, and $\mathrm{CD} 3^{+} \mathrm{CD} 8^{+}$IFN- $\gamma^{+}$sets of T lymphocytes by flow cytometry. Results of separate experiments are shown in Supplemental Figure S3. B: Representative dot-plot images of 7-day-infected mice, emphasizing the gating strategy for quantification of different T-lymphocyte sets. C: The geometric mean fluorescence intensity of intracellularly expressed interferon (IFN)- $\gamma$ protein in BALF CD3 ${ }^{+} \mathrm{CD} 8^{+}$IFN- $\gamma^{+}$T lymphocytes during infection was analyzed by flow cytometry. The fluorescence intensity of noninfected WT CD3 ${ }^{+} \mathrm{CD} 8^{+}$IFN- $\gamma^{+}$cells was assigned a value of 1 . Values of individual animals and mean values (black lines) per group are shown. $n=2$ independently repeated experiments. Statistical analysis was performed with the $U$-test. ${ }^{*} P<0.05,{ }^{* *} P<0.01$, and ${ }^{* * *} P<0.001$.

lymphocytes was significantly higher in $F H L 2^{K O}$ than in WT mice on infection, even though the virus replicated to a significantly lesser extent in the lungs of $F H L 2^{K O}$ mice. This was clearly seen on 7 day after infection, at the time when the adaptive T-cell response was well unforced, but also on day 3 after infection when the T-cell response just started to expand, a tendency of a higher response was present, especially for the activated $\mathrm{CD} 8^{+} \mathrm{IFN}-\gamma^{+} \mathrm{T}$ cells (Figure $3 \mathrm{~A}$ ). The degree of $\mathrm{CD}^{+}{ }^{+} \mathrm{CD} 8{ }^{+}$lymphocyte activation measured by relative induction of intracellular IFN- $\gamma$, however, was equal in both
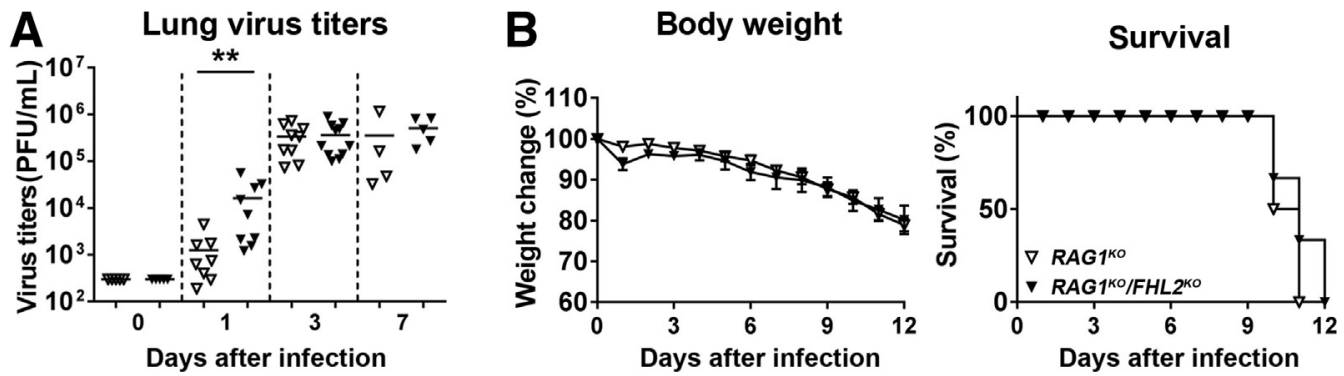



Days after infection

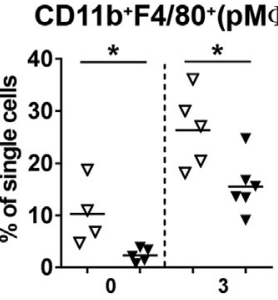

Days after infection

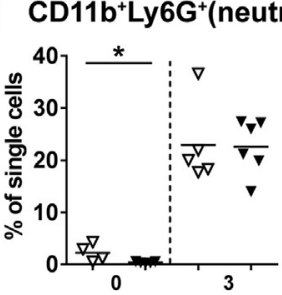

Days after infection
CD3-CD49b+IFN $\gamma^{+}$

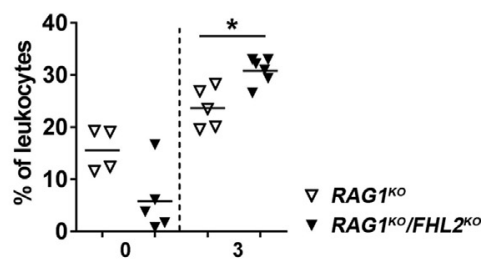

Days after infection

Figure 4 Deficiency in four-and-a-half LIM domain protein 2 (FHL2) does not accelerate influenza A viral clearance in the absence of the adaptive immune system. A: $R A G 1^{K O}$ and $R A G 1^{K O} / F H L 2^{K O}$ mice were infected with 300 plaque-forming units (PFU; $1 \times L_{50}$ ) of influenza A/PR/8/34 (PR8) H1N1 strain and at indicated times, virus titers in bronchoalveolar lavage fluid (BALF) were determined by standard plaque assay. B: Body weight and survival of PR8-infected $R A G 1^{K O}$ and $R A G 1^{K O} / F H L 2^{K O}$ mice were determined daily. Mice of both lineages were sacrificed at days 10 to 12 of infection because of ethical criteria. C: BALF of noninfected or 3-day-long infected $R A G 1^{K O}$ and $R A G 1^{K O} / F H L 2^{K O}$ mice were analyzed for alveolar macrophages (aM ${ } ; \mathrm{CD}_{11 \mathrm{c}^{+}} \mathrm{SigF}^{+}$), peripheral macrophages

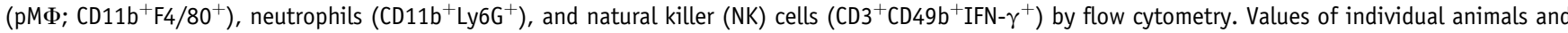
mean values (black lines) per group are shown. $n=2$ independent experiments $(\mathbf{A})$. Statistical analysis was performed with the $U$-test. ${ }^{*} P<0.05$, ${ }^{* *} P<0.01$. IFN, interferon; K0, knockout. 
mouse strains (Figure 3C), suggesting that only the number but not the activity of anti-influenza cytotoxic $\mathrm{T}$ cells was increased in FHL2-deficient mice.

To verify whether the faster IAV clearance in $F H L 2^{K O}$ mice in comparison with WT mice was indeed induced by the increased adaptive immune response in these animals, $F H L 2^{K O}$ mice were crossed with $R A G 1^{K O}$ mice that lacked mature $\mathrm{T}$ and $\mathrm{B}$ lymphocytes, and the double mutants were analyzed for viral replication in comparison with $R A G 1^{K O}$ only mutants (Figure 4A). Similar to $F H L 2^{K O}$ mice with $R A G 1$ WT background, the $F H L 2^{K O} / R A G 1^{K O}$ mice displayed a higher virus yield at early time of infection compared with $R A G 1^{K O}$ only mutants. However, on days 3 and 7 of infection, both mouse strains showed a comparable viral load. In agreement with this, the body weight change and survival rates of IAV-infected $R A G 1^{K O}$ and $F H L 2^{K O} / R A G 1^{K O}$ mice were similar and did not reflect the kinetics of WT and $F H L 2^{K O}$ mice (Figure 4B). These data, together with the development of the innate host immune response in $R A G 1^{K O}$ and $F H L 2^{K O} / R A G 1^{K O}$ mice (Figure $4 \mathrm{C}$ ), suggested that the switch in the efficiency of viral replication seen in $F H L 2^{K O}$ mice in relation to WT animals was indeed based on a stronger adaptive immune response in FHL2-deficient mice.

\section{Discussion}

Here, we demonstrate that IAVs replicate significantly better in lungs of $F H L 2^{K O}$ mice than in WT mice, but only at early stages of infection. With ongoing infection, the viral replication in $F H L 2^{K O}$ lungs is delayed in comparison with WT lungs. We further show that the enhanced clearance of viral infection in FHL2 KO mice correlates with increased recruitment of IFN- $\gamma$-expressing $\mathrm{CD} 8^{+} \mathrm{T}$ lymphocytes into infected lungs and with accelerated migration of FHL2deficient DC subsets to the proximal LNs.

As an adaptor protein, FHL2 is involved in regulation of different signaling pathways and gene transcription. It interacts with integrins, kinases, structural proteins, and transcription factors, thereby modulating different signaling cascades and cellular processes. ${ }^{10,11}$ Depending on the cellular and molecular background or interaction partners, FHL2 may even exert opposing functions, ${ }^{10,11}$ a common characteristic for many adaptor proteins. ${ }^{33}$ For instance, by binding to $\beta$ catenin, FHL2 inhibits Lef/Tcf-mediated transcription and proliferation in myocytes, ${ }^{34}$ but it enhances proliferation in several malignant tumor cell lines. ${ }^{35}$ By binding to integrin receptors and the focal adhesion kinase, FHL2 participates in activation of Rac GTPases and in inhibition of extracellular signal-related kinase mitogen-activated protein kinases, leading to alterations in cytoskeleton and extracellular matrix assembly and to migration support of mesenchymal cells. ${ }^{16,17}$ In DCs, to the contrary, FHL2 inhibits Rac activation by repression of S1PR1 expression, causing reduced motility of these cells ${ }^{15}$ (this work) and concomitantly affecting the outcome of pathogenic infections. Thus, highly dependent on the background, FHL2 executes ambiguous functions. The comparative kinetics of the viral load in WT and $F H L 2^{K O}$ lungs demonstrated the dual mode of FHL2 action at best. Although FHL2 protected epithelial cells against influenza viral infection by strengthening the innate cellular immune response,$^{20}$ it supported at the same time a sustained viral replication in lungs at later phases of infection by impeding the adaptive immune response and DC migration to the LNs (this work).

After lung infection, IAVs have to overcome at least three highly efficient immune defense barriers: the intrinsic cellular innate response, the host innate immune response, and the host adaptive immune response. ${ }^{25,26}$ The first response takes effect in a few hours after infection, reaches its peak during the first 24 hours, and declines afterward. This line of defense is rather unspecific and is represented by production of the type I IFN molecules that in turn induce expression of IFN-responsive genes such as myxovirus resistance gene $1(M x l), 2^{\prime}, 5^{\prime}$-oligoadenylate synthetase $1(O A S 1)$, or protein kinase $\mathrm{R}$ (PKR, official name Eif2ak2), which restrain virus propagation by suppression of cellular transcription and translation activities. ${ }^{7,36}$ Besides this, type I IFNs also participate in sensitization of the host immune defense. ${ }^{6}$ The second defense barrier rendered by effector cells such as macrophages, neutrophils, NK cells, and antigen-presenting DCs leads to lysis of infected cells and antigen transmission. This response begins shortly after infection and reaches its maximal activity within a few days. ${ }^{26,37}$ It is also rather unspecific and might affect a broad range of pathogens. The third type of antiviral defense, the adaptive immune response, results in development of high numbers of cytotoxic $\mathrm{T}$ lymphocytes and antibodyproducing B lymphocytes. This type of immune defense is highly specific and effective. It unfolds its strength only with time and reaches the maximum when only a few or no viruses are detectable in the lung. ${ }^{26,38,39}$ Nevertheless, the adaptive immune response is decisive for clearing the viral infection and mouse survival, and RAG KO mice lacking mature $\mathrm{T}$ and $\mathrm{B}$ lymphocytes are not able to clear viral infections and die being infected with IAV doses at which all WT mice were still able to survive. ${ }^{40}$

In the present study, a differential weighting of immune defense barriers in clearance of IAV infection was observed. The in vitro data showed that expression of FHL2 confers a more than 10-fold higher protection of epithelial cells against viral infection compared with FHL2-deficient cells, just because of the support of the cellular innate immune response. FHL2 is enhancing the IRF-3-mediated transcription of the Ifnb1 gene. ${ }^{20}$ This activity of FHL2 was also beneficial for IAV-infected mice. It suppressed influenza virus replication in lungs of WT mice, but only for a short time early in infection. Indeed, 1 day after infection, WT lungs revealed a significantly lower viral load than $F H L 2^{K O}$ lungs. However, only 2 days later, the initial advantage the WT mice had over $F H L 2^{K O}$ mice swapped into a drawback. In the absence of FHL2 the replication rate of influenza 
viruses significantly decreased and $F H L 2^{K O}$ mice successfully cleared the viral infection, whereas WT mice could not recover after infection with the same viral dose (Figure 1). The reason for the radical change in the resistance to influenza virus spread in $F H L 2^{K O}$ mice was the upcoming adaptive immune response, represented by $\mathrm{CD} 3{ }^{+} \mathrm{CD} 8{ }^{+}$IFN$\gamma^{+} \mathrm{T}$ lymphocytes. The relative number of these cells was already higher on day 3 after infection in $F H L 2^{K O}$ mice than in WT mice and increased further on day 7 after infection (Figure 3). Phenotypically, this led to a more efficient clearance and a lower pathologic severeness of infection. The central role of $\mathrm{T}$ lymphocytes in improved viral clearance in $F H L 2^{K O}$ mice was further validated by infecting $R A G 1^{K O}$ mice. Lungs of double-mutated $R A G 1^{K O} / F H L 2^{K O}$ mice displayed on day 1 of infection, similar to $F H L 2^{K O}$ mice, increased numbers of IAV particles in comparison with their FHL2-containing WT counterparts, but did not differ in viral titers from $R A G I^{K O}$ mice at later stages of infection as well as in body weight changes and survival rates after infection. These data strongly support the hypothesis that the elevated CD8 T-cell response in FHL2 ${ }^{K O}$ mice was decisive for their higher resistance to influenza virus infection. Thus, it seems that the boost of the third defense barrier to IAV infection competed out the beneficial effect the influenza viruses originally had in $F H L 2^{K O}$ mice because of the weak first barrier of cell intrinsic innate immune response. In WT mice, the increased antiviral state provided by FHL2 through enhanced induction of type I IFNs was not sufficient to successfully maintain a long-term antiviral barrier in infected lungs, even in combination with present host innate and later upcoming host adaptive immune responses. Hence, the balance between the three lines of immune defense and the initial pathogen load matters significantly for the outcome of viral respiratory infections with IAVs.

\section{Conclusion}

This analysis of the impact of FHL2 on IAV replication unveiled opposing actions of the protein on different antiviral defense barriers. Although FHL2 reduced viral replication at early phases of lung infection by supporting type I IFN expression in lung epithelial cells, it promoted influenza virus replication at later stages of lung infection, probably by reducing migration of antigen-presenting $\mathrm{CD} 11 \mathrm{c}^{+} \mathrm{CD} 103^{+}$ $\mathrm{CD} 11 \mathrm{~b}^{-}$DCs from the site of inflammation to the regional LNs and infiltration of infected lungs with cytotoxic $\mathrm{CD}^{+} \mathrm{CD}^{+}$ IFN- $\gamma^{+} \mathrm{T}$ lymphocytes.

\section{Acknowledgment}

Goat anti-influenza NP antibody (clone G150) was provided by Dr. Robert Webster (St. Jude Children's Research Hospital, Memphis, TN).

\section{Supplemental Data}

Supplemental material for this article can be found at https://doi.org/10.1016/j.ajpath.2018.02.004.

\section{References}

1. Ludwig S, Pleschka S, Planz O, Wolff T: Ringing the alarm bells: signalling and apoptosis in influenza virus infected cells. Cell Microbiol 2006, 8:375-386

2. Ludwig S, Zell R, Schwemmle M, Herold S: Influenza, a One Health paradigm-novel therapeutic strategies to fight a zoonotic pathogen with pandemic potential. Int J Med Microbiol 2014, 304:894-901

3. Peteranderl C, Herold S, Schmoldt C: Human influenza virus in fections. Semin Respir Crit Care Med 2016, 37:487-500

4. Chakrabarti AK, Pasricha G: An insight into the PB1F2 protein and its multifunctional role in enhancing the pathogenicity of the influenza A viruses. Virology 2013, 440:97-104

5. Krug RM: Functions of the influenza A virus NS1 protein in antiviral defense. Curr Opin Virol 2015, 12:1-6

6. Kawai T, Akira S: Innate immune recognition of viral infection. Nat Immunol 2006, 7:131-137

7. Ehrhardt C, Seyer R, Hrincius ER, Eierhoff T, Wolff T, Ludwig S: Interplay between influenza A virus and the innate immune signaling. Microbes Infect 2010, 12:81-87

8. GeurtsvanKessel CH, Willart MA, van Rijt LS, Muskens F, Kool M, Baas C, Thielemans K, Bennett C, Clausen BE, Hoogsteden HC, Osterhaus AD, Rimmelzwaan GF, Lambrecht BN: Clearance of influenza virus from the lung depends on migratory langerin $+\mathrm{CD} 11 \mathrm{~b}-$ but not plasmacytoid dendritic cells. J Exp Med 2008, 205:1621-1634

9. Herold S, Mayer K, Lohmeyer J: Acute lung injury: how macrophages orchestrate resolution of inflammation and tissue repair. Front Immunol 2011, 2:65

10. Johannessen M, Moller S, Hansen T, Moens U, Van Ghelue M: The multifunctional roles of the four-and-a-half-LIM only protein FHL2 Cell Mol Life Sci 2006, 63:268-284

11. Kleiber K, Strebhardt K, Martin BT: The biological relevance of FHL2 in tumour cells and its role as a putative cancer target. Anticancer Res 2007, 27:55-61

12. Alnajar A, Nordhoff C, Schied T, Chiquet-Ehrismann R, Loser K, Vogl T, Ludwig S, Wixler V: The LIM-only protein FHL2 attenuates lung inflammation during bleomycin-induced fibrosis. PLoS One 2013, 8:e81356

13. Bai S, Zha J, Zhao H, Ross FP, Teitelbaum SL: Tumor necrosis factor receptor-associated factor 6 is an intranuclear transcriptional coactivator in osteoclasts. J Biol Chem 2008, 283:30861-30867

14. Dahan J, Nouet Y, Jouvion G, Levillayer F, Adib-Conquy M, CassardDoulcier AM, Tebbi A, Blanc F, Remy L, Chen J, Cairo S, Werts C, Si-Tahar M, Tordjmann T, Buendia MA, Wei Y: LIM-only protein FHL2 activates NF-kappaB signaling in the control of liver regeneration and hepatocarcinogenesis. Mol Cell Biol 2013, 33:3299-3308

15. Konig K, Diehl L, Rommerscheidt-Fuss U, Golletz C, Quast T, Kahl P, Kolanus W, Knolle P, Buettner R, Heukamp LC: Four-and-a-half LIM domain protein 2 is a novel regulator of sphingosine 1-phosphate receptor 1 in CCL19-induced dendritic cell migration. J Immunol 2010, 185:1466-1475

16. Park J, Will C, Martin B, Gullotti L, Friedrichs N, Buettner R, Schneider H, Ludwig S, Wixler V: Deficiency in the LIM-only protein FHL2 impairs assembly of extracellular matrix proteins. FASEB J 2008, 22:2508-2520

17. Wixler V, Hirner S, Muller JM, Gullotti L, Will C, Kirfel J, Gunther T, Schneider H, Bosserhoff A, Schorle H, Park J, Schule R, Buettner R: Deficiency in the LIM-only protein Fhl2 impairs skin wound healing. J Cell Biol 2007, 177:163-172 
18. Leite Dantas R, Brachvogel B, Schied T, Bergmeier V, Skryabin B, Vogl T, Ludwig S, Wixler V: The LIM-only protein four and a half LIM domain protein 2 attenuates development of psoriatic arthritis by blocking Adam17-mediated tumor necrosis factor release. Am J Pathol 2017, 187:2388-2398

19. Wixler V, Cromme C, Retser E, Meyer LH, Smyth N, Muhlenberg K, Korb-Pap A, Koers-Wunrau C, Sotsios Y, Bassel-Duby R, Baeten D, Tak PP, Niederreiter B, Redlich K, Bertrand J, Skryabin BV, Ludwig S, Pap T: FHL2 regulates the resolution of tissue damage in chronic inflammatory arthritis. Ann Rheum Dis 2015, 74:2216-2223

20. Nordhoff C, Hillesheim A, Walter BM, Haasbach E, Planz O, Ehrhardt C, Ludwig S, Wixler V: The adaptor protein FHL2 enhances the cellular innate immune response to influenza A virus infection. Cell Microbiol 2012, 14:1135-1147

21. Kong Y, Shelton JM, Rothermel B, Li X, Richardson JA, BasselDuby R, Williams RS: Cardiac-specific LIM protein FHL2 modifies the hypertrophic response to beta-adrenergic stimulation. Circulation 2001, 103:2731-2738

22. Dudek SE, Wixler L, Nordhoff C, Nordmann A, Anhlan D, Wixler V, Ludwig S: The influenza virus PB1-F2 protein has interferon antagonistic activity. Biol Chem 2011, 392:1135-1144

23. Borgeling Y, Schmolke M, Viemann D, Nordhoff C, Roth J, Ludwig S: Inhibition of p38 mitogen-activated protein kinase impairs influenza virus-induced primary and secondary host gene responses and protects mice from lethal H5N1 infection. J Biol Chem 2014, 289:13-27

24. Kother K, Nordhoff C, Masemann D, Varga G, Bream JH, Gaestel M, Wixler V, Ludwig S: MAPKAP kinase 3 suppresses Ifng gene expression and attenuates NK cell cytotoxicity and Th1 CD4 T-cell development upon influenza A virus infection. FASEB J 2014, 28 : 4235-4246

25. Openshaw PJ, Tregoning JS: Immune responses and disease enhancement during respiratory syncytial virus infection. Clin Microbiol Rev 2005, 18:541-555

26. Pommerenke C, Wilk E, Srivastava B, Schulze A, Novoselova N, Geffers R, Schughart K: Global transcriptome analysis in influenzainfected mouse lungs reveals the kinetics of innate and adaptive host immune responses. PLoS One 2012, 7:e41169

27. Hussell T, Bell TJ: Alveolar macrophages: plasticity in a tissue-specific context. Nat Rev Immunol 2014, 14:81-93
28. Ghoneim HE, Thomas PG, McCullers JA: Depletion of alveolar macrophages during influenza infection facilitates bacterial superinfections. J Immunol 2013, 191:1250-1259

29. Ballesteros-Tato A, Leon B, Lund FE, Randall TD: Temporal changes in dendritic cell subsets, cross-priming and costimulation via CD70 control CD8(+) T cell responses to influenza. Nat Immunol 2010, 11: 216-224

30. Legge KL, Braciale TJ: Accelerated migration of respiratory dendritic cells to the regional lymph nodes is limited to the early phase of pulmonary infection. Immunity 2003, 18:265-277

31. Forster R, Davalos-Misslitz AC, Rot A: CCR7 and its ligands: balancing immunity and tolerance. Nat Rev Immunol 2008, 8: 362-371

32. Liu M, Zhao X, Hua S, Du X, Peng Y, Li X, Lan Y, Wang D, Wu A, Shu Y, Jiang T: Antigenic patterns and evolution of the human influenza A (H1N1) virus. Sci Rep 2015, 5:14171

33. Ayllon J, Garcia-Sastre A: The NS1 protein: a multitasking virulence factor. Curr Top Microbiol Immunol 2015, 386:73-107

34. Martin B, Schneider R, Janetzky S, Waibler Z, Pandur P, Kuhl M, Behrens J, von der Mark K, Starzinski-Powitz A, Wixler V: The LIMonly protein FHL2 interacts with beta-catenin and promotes differentiation of mouse myoblasts. J Cell Biol 2002, 159:113-122

35. Wei Y, Renard CA, Labalette C, Wu Y, Levy L, Neuveut C, Prieur X, Flajolet M, Prigent S, Buendia MA: Identification of the LIM protein FHL2 as a coactivator of beta-catenin. J Biol Chem 2003, 278:5188-5194

36. Durbin RK, Kotenko SV, Durbin JE: Interferon induction and function at the mucosal surface. Immunol Rev 2013, 255:25-39

37. Herold S, Becker C, Ridge KM, Budinger GR: Influenza virus-induced lung injury: pathogenesis and implications for treatment. Eur Respir J 2015, 45:1463-1478

38. Grant EJ, Quinones-Parra SM, Clemens EB, Kedzierska K: Human influenza viruses and CD8(+) T cell responses. Curr Opin Virol 2016, $16: 132-142$

39. Newton AH, Cardani A, Braciale TJ: The host immune response in respiratory virus infection: balancing virus clearance and immunopathology. Semin Immunopathol 2016, 38:471-482

40. Wu H, Haist V, Baumgartner W, Schughart K: Sustained viral load and late death in Rag2-/- mice after influenza A virus infection. Virol J 2010, 7:172-177 